\title{
East Industrial Pedestrian Bridge: A Case Studyof Value Engineering and High Performance Material
}

\author{
Theodoros Tzaveas \\ ttzaveas@ashghal.gov.qa \\ Public Works Authority (Ashghal), Doha, Qatar
}

\begin{abstract}
East Industrial Road, a main arterial for the city of Doha with high average daily traffic, made crossing by foot being not only challenging, but also dangerous. Authorities proposed to build a pedestrian bridge over East Industrial Road which would allow for easy, safe travel over this busy roadway. As part of initiatives towards reduced cost of road construction and maintenance, alternative materials for the bridge were considered. For the construction phase, quick installation and utilization of prefabricated units, and for the operational phase, solutions not requiring periodical maintenance were favored. The primary objective of this paper is to describe the potential of aluminum in competition with common steel solutions for this pedestrian bridge. Collaborative design work remains a key aspect in projects that addresses not only technical efficiency but also human experience. Cross-disciplinary collaboration requires a language that allows exchanges across disciplinary boundaries. This paper also discusses the design process of the first pedestrian bridge built in Qatar made of structural aluminum. As engineering and building challenges increase in complexity due to environmental and political factors inherent in the modern age, as well as ever accelerating changes in technology, project clients are seeking ways for effective management. The purpose of value engineering is to improve the value of the project designs and to demonstrate to the client that the objective of "value" has been achieved. The process is documented to illustrate the range of considerations required in the design/construction team to ensure a successful project implementation.
\end{abstract}

Keywords: Pedestrian bridge; Structural aluminum; Aerodynamic effects; Vibration control; Value engineering

\section{INTRODUCTION}

In 2013, Public Works Authority (PWA) developed a program to deliver gradeseparated pedestrian bridges in different parts of Qatar to provide a complete separation of pedestrian and vehicular traffic and make it easier and safer for pedestrians to walk to a variety of destinations such as schools, shopping centers and public transport.

Early 2014 the program was launched, initiating a procurement phase for highpriority locations mostly in terms of pedestrian safety based on traffic fatality figures. From the priority list, a specific location at East Industrial Road was selected for the first pedestrian bridge to be built by PWA. Value engineering principles applied to produce meaningful results within a reasonable schedule encouraging owner, contractor and stakeholder participation in the assessment in order to take advantage of experience and knowledge, which significantly increases the value of the ideas presented, and the 
implementation of recommendations. In August 2014, PWA awarded a design \& built contract to a joint venture of specialized contractors for the design and construction of an aluminum pedestrian bridge across the East Industrial Road. Work on site was completed late in the spring of 2015. This paper will demonstrate how the design has been developed specifically for its context; how it overcomes the challenging site constraints, the conflict between the design aspirations for a fully enclosed, air conditioned corridor, limited site access, and clearance constraints, and key design constraints for quick installation and utilization of prefabricated units, not requiring periodical maintenance for the operational phase while enhancing connectivity between the two development sites on either side of the road. Wind tunnel testing was required to demonstrate aerodynamic stability of the footbridge and detailed vibration computations carried out to ensure comfort levels to end users. The pedestrian counter installed on the bridge justifies the need and significant use of the bridge.

\section{SITE}

The bridge is located in the Industrial Area of Doha, on East Industrial Road at about chainage $123+560$ along the main carriageway. East Industrial Road is a busy expressway serving the industrial area. There is a high percentage of HGVs along the route. It is a dual carriageway with a narrow median and the mainline consists of three (3) lanes in both directions. The service roads are 2 lanes. The industrial area is located to the south of East Industrial Road and commercial and residential areas are located to the north of road. Considering the development of the area, pedestrian desire lines were clearly defined between these areas that the bridge would ultimately serve.

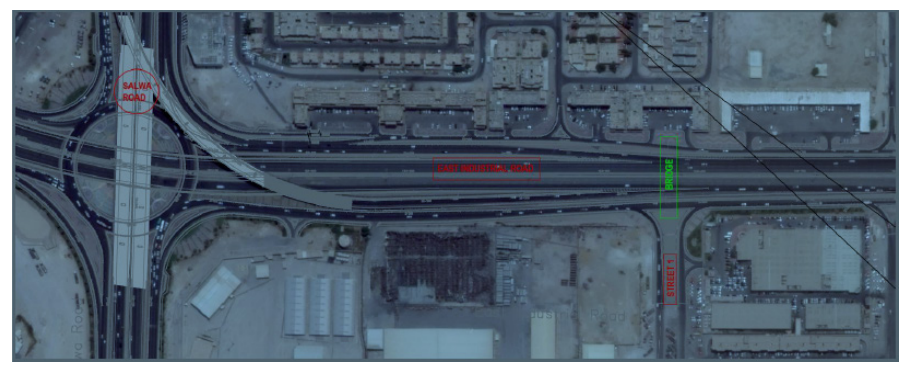

Figure 1: Location map with the position of the bridge (in green)

\section{VALUE ENGINEERING (VE)}

Projects tend to be initiated based on linear, generic thinking that mostly consider the primary functions. For the particular project, additional requirements and constraints were merged as the concept was developing. Value engineering takes a step back and looks at the project from a functional perspective rather than how it is originally conceived. VE helps organizations improve the delivery of a project or product by reducing cost, improving performance, improving quality, saving time during construction, solving problems and using resources effectively.

Value considerations are not necessarily priorities in the planning of a project when compared with project cost analysis. Often, the design effort does not address life cycle cost. Scoping constraints, design standards and stakeholder demands often override any 
initiative in the planning process to optimize the design or to manage costs. Often there is little time in the planning process for creativity and innovation in design. The risk associated with project delivery, schedule and operations may not be apparent in the project planning phase. For these reasons, lowest price option is not always the best value solution, which can be achieved in design. The project team reviewed the project functions and identified key components, as listed below:

- Design standards.

- Space for assembly but limited access and maneuvering.

- Congested area with services and utilities - limited scope for diversions.

- Continuous (\& heavy) traffic 24/7.

- Limited space for staircase landings.

- Foundation footprint shall be limited to median.

- Limited road closures.

- High durability demand due to environmental exposure conditions.

- Options for dismantling and re-usability.

- Minimum inspection and maintenance requirements.

- Enclosed deck - Air Conditioned.

- Elevators.

- CCTV, fire safety, internal/external lighting.

Options comprising both steel and aluminum were assessed against an initial baseline concept of an open steel bridge configuration originally proposed for the particular site. Performance criteria were established as a basis of comparing options including safety, quality, aesthetics, new technologies, constructability, convenience, schedule and whole life cost. At the end of the process, a modular type aluminum enclosed truss as illustrated in Figure 2 was the preferred option to be developed to a detailed design.

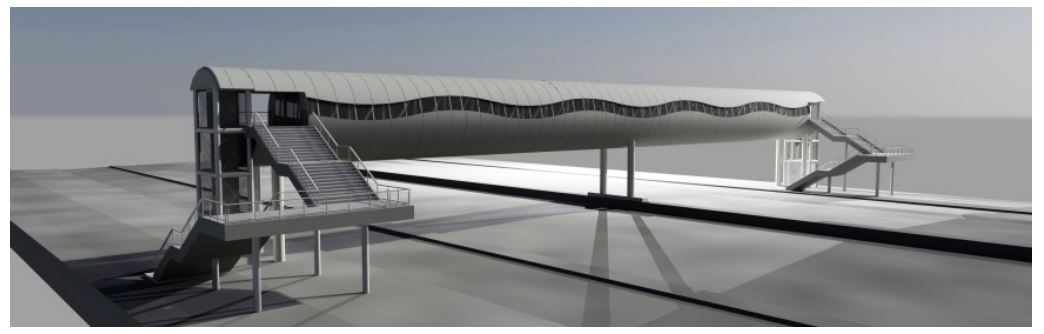

Figure 2: Rendering of the preferred option in aluminum

\section{FOOTBRIDGE FORM AND DEVELOPMENT OF DESIGN}

The proposed footbridge is a two span aluminum bridge with a total length of approximately $84 \mathrm{~m}$. The bridge is located within the right of way in the industrial area providing a safe crossing point over the East Industrial Road. The deck is enclosed with a cladding made of $4 \mathrm{~mm}$ thick aluminum panels attached to a circular aluminum frame with double-glazing on the sides to provide the necessary transparency while maintaining air conditioning and ventilation of the enclosed footbridge deck. Access onto the bridge is provided by a staircase and lifts at both ends. The footbridge is equipped with air condition and ventilation system, CCTV, lighting both inside and outside, fire alarm and fire extinguishing devices, and a counter device which together with the lifts, are all 
integrated into a Building Management System (BMS) to allow various types of control during the operation phase.

\subsection{Concept design}

The bridge comprised of a pre-engineered aluminum (Howe) trusses consisting of two simply supported spans measuring $40.15 \mathrm{~m} \& 38.15 \mathrm{~m}$. An expansion joint is introduced only at the middle over the central pier as illustrated in Figure 3. The pre-engineered solution developed further to improve the stiffness and capacity to accommodate the added loads as a result of the enclosures as well as adhering to deflection requirements of L/400. The bridge is designed to satisfy the requirements of BS5400-DMRBs, BS EN 1991-2 \& 1999 and AASHTO LRFD. Adherence to PWA Interim Advice Notes 009 (Design Criteria for Highway Structures) and 006 (Specification for Bridge Bearings) and QCS are ensured through the various design phases.

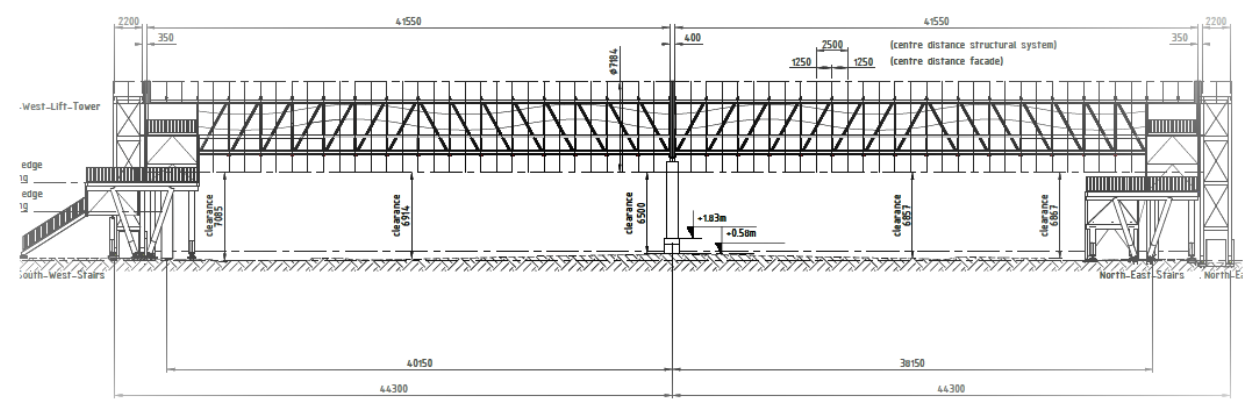

Figure 3: Elevation and span arrangement

\subsection{Superstructure}

The trusses are $4.80 \mathrm{~m}$ high and spaced at $4.75 \mathrm{~m}$. The overall height of the bridge including the cladding is $7.18 \mathrm{~m}$. Effective deck width for the movement of pedestrians is $4.00 \mathrm{~m}$ with a total width of $6.55 \mathrm{~m}$ to include the maintenance corridors for access into the enclosure. Figure 4 illustrates the details of a typical truss.

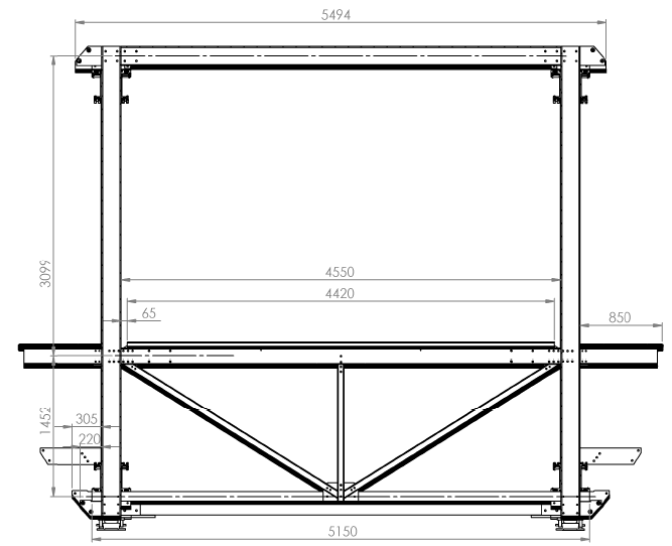

Figure 4: Cross section of typical truss 
One of the big advantages of aluminum when compared to steel is ease of forming and the possibility of extruding complex profiles. This gives the possibility to design a multi-functional profile without extra costs. Truss members comprise of open channel section for the truss chords, hollow sections for the transverse frames and the diagonal members and T-sections for the cladding frames all made of alloy EN-AW 6082 T6 with an ultimate strength of $310 \mathrm{Mpa}$. All connections between aluminum members are bolted with stainless steel bolts of grade A4-70 to BS EN ISO 3506-1. When significant contact areas between dissimilar metals present in a joint location insulating washers or pads are introduced to isolate the different metals, aluminum extruded deck panels, $50 \mathrm{~mm}$ thick, with a roughened surface oriented perpendicular to the traffic direction and the top of the transverse frames act as the walking surface.

\subsection{Foundations and substructures}

The exploratory holes conducted at support locations indicated that the subsurface stratigraphy is generally consisting of quaternary deposits and generally consists of slightly dense light brown, slightly silty, very sandy fine to coarse gravel of limestone. The Simsima limestone was encountered in all boreholes and generally consists of weak to medium strong, very thickly bedded, light grey, fine grained dolomitic limestone with few voids and few inclusions of very weak light greenish grey fine grained calcareous siltstone. Due to the lightweight features of the superstructure, it was possible to optimize the foundation footprint. The bridge supports consist of twin circular concrete columns with a diameter of $0.90 \mathrm{~m}$ resting on rectangular concrete footings, with plan dimensions $10 \times 1.6 \mathrm{~m}$ in the median and 10x1.8m at the two ends. With these optimized sizes impact, it was made possible to get around existing/future utilities corridors and minimize disruption during the excavation in the median.

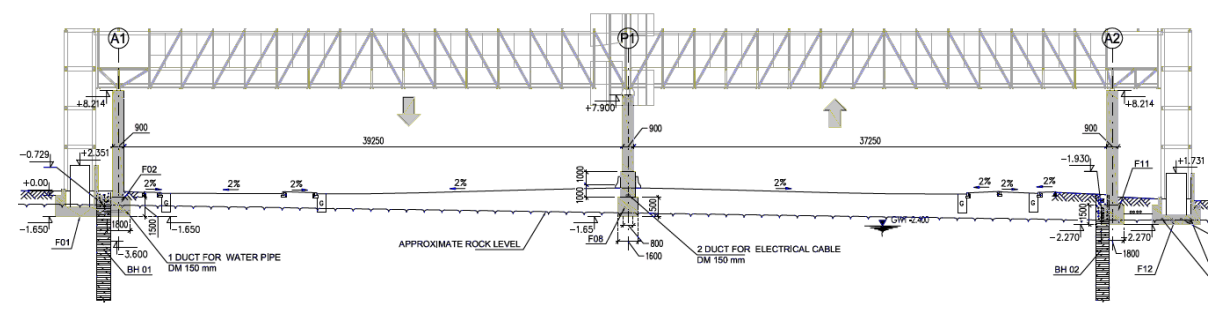

Figure 5: Foundation configurations

\subsection{Articulation}

The aluminum deck is supported on the columns through spherical bearings. Both spans are restrained transversally, and only allowed to slide longitudinally over the middle support. To accommodate the movements in the middle, both columns have a steel bracket to efficiently provide space for the pair of bearings for each span as illustrated in Figure 6. 


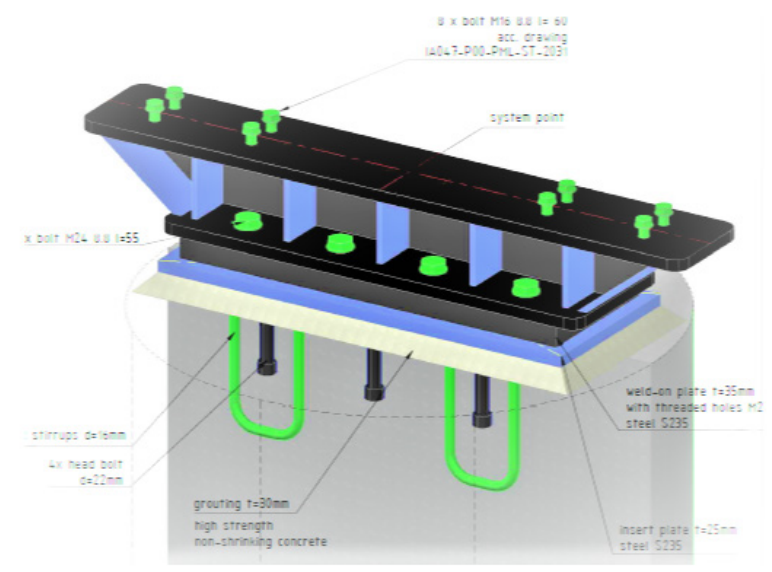

Figure 6: Steel brackets on intermediate piers

\subsection{Aerodynamic assessment}

The aerodynamic Susceptibility Parameter $(\mathrm{Pb})$ was calculated as per BD 49 and resulted in a value of 0.26 , which is within the scope of the rules specified in BD 49 . Assessment of the bridge against the given criteria and limitations, suggested that, whilst the footbridge satisfies the limits for vortex excitation, but it could not comply with the divergent amplitude response checks for galloping and stall flutter. It was therefore necessary to demonstrate by other means that the wind speed required to induce the onset of galloping was in excess of the critical wind speed limit (calculated as $53 \mathrm{~m} / \mathrm{s}$ ). A wind tunnel test illustrated in Figure 7 was subsequently commissioned and the footbridge was found to have sufficient stability against galloping and flutter. Resonant vortex shedding effects were excluded within the range of expected wind speeds at project site.

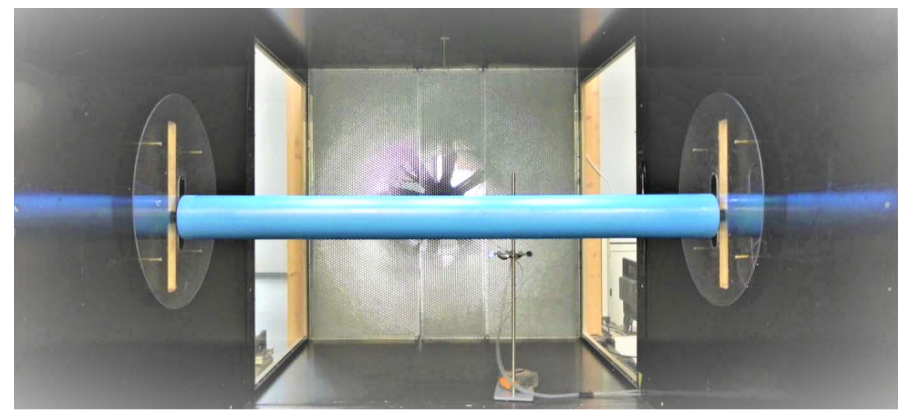

Figure 7: Section model in the wind tunnel test

\subsection{Vibrations}

The footbridge was also checked for vibration serviceability to ensure that the structure was not excessively excited by pedestrian use in reference to BD37 and BS EN 1991-2. The determination of natural frequencies and analysis of induced vibrations was made by a special software using 3D model as shown in Figure 8 . 


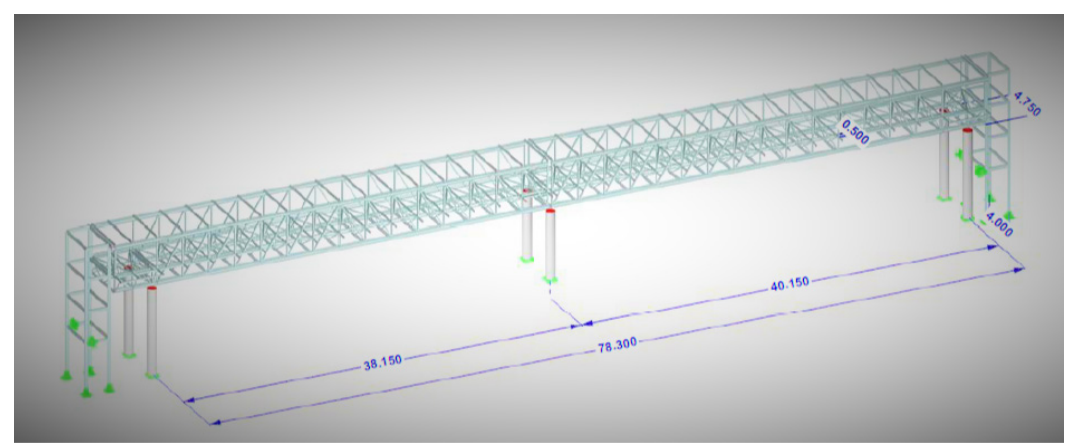

Figure 8: Isometric view of analytical model

In vertical direction the fundamental natural frequency was found to be $2.23 \mathrm{~Hz}$. Therefore, maximum vertical acceleration had to be assessed. The assessment based on the provisions of NA to BS EN 1991-2 and carried out for walking and crowded, resulted in values of $0.585 \mathrm{~m} / \mathrm{s} 2$ and $1.485 \mathrm{~m} / \mathrm{s} 2$ respectively. When compared with the maximum design acceleration limit of $2.0 \mathrm{~m} / \mathrm{s} 2$ was found satisfactory. The natural frequency in horizontal direction satisfies the threshold of $1.50 \mathrm{~Hz}$.

\section{CONSTRUCTION}

Adopting a configuration with bolted connections and manageable lightweight member lengths may pose some difficulties and risks during the assembly process but on the other hand proved to be advantageous in the construction logistics and the delivery schedule. Aluminum was fabricated off site in fabrications workshops overseas under well controlled and monitored conditions. The members delivered to site section sizes suitable to be transported in standard container sizes. The area adjacent to the bridge site was made available to the construction team for assembly of the bridge. This removed significant health and safety risks associated with erecting and assembling large bridge modules and transporting them to the bridge site. Carriageway possessions for footbridge erection and construction were clearly very limited for such a busy corridor. The reduced weight that the aluminum offers, allowed spans to be constructed progressively, including cladding and finishes supported on temporary trestles and then positioned on the piers during only two overnight possessions using a pair of mobile cranes for each span. Figure 9 shows a typical span being assembled including the cladding and subsequently erected using mobile cranes. When the $41 \mathrm{~m}$ long span module was lifted for installation it was weighing approximately 24 tons.

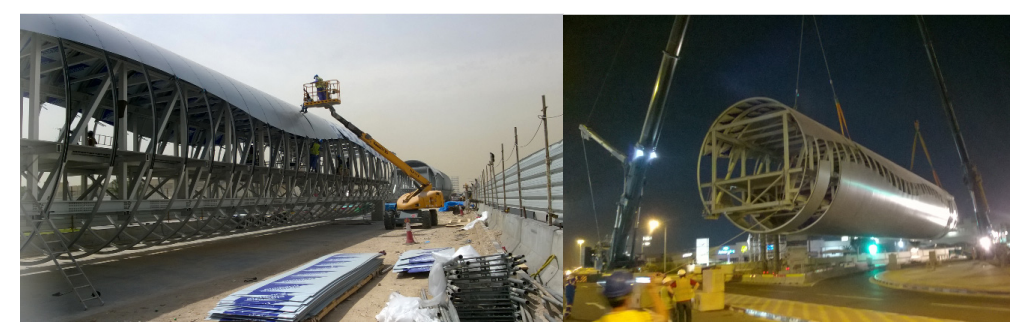

Figure 9: Off-site assembly and erection of typical span 


\section{CONCLUSION}

The design and construction of the East Industrial Pedestrian Bridge, from inception to final handover was challenging. End users prove the success of the project when considering the recorded number of passages over the bridge which was just over two (2) million by the end of 2018. By applying value engineering principles even in a slightly informal way opened the opportunities for non-conventional approaches to be adopted and new materials to be utilized. The primary objective of this paper is to present the potential of aluminum solutions within pedestrian bridges based on a case study in the State of Qatar. As a construction material, aluminum contains several advantages; high specific strength, high corrosion resistance, and no need of periodic maintenance. Concerning manufacturability of aluminum, smart and creative use of manufacturing methods and pre-engineered solutions give the material an advantage to implement a tight design and construction programme.

Communication is paramount to handling the challenges on a project of this scope and magnitude. The project team is large, the scope of work often widens to adopt client's aspirations and stakeholder requirements, things happen fast, and everything must get underway with a minimum of lead-time. Effective communication mostly comes from hard work, interpersonal interaction and controls guiding the conduct of the communication process throughout the project. Understanding this and the principals that govern effective communication the project team collaborated with the construction team to develop an effective communication path between the designer, the independent checker, the contractor and the subcontractors and material suppliers which resulted in the successful implementation of the project.

\section{REFERENCES}

British Standards Institution. Eurocode 9: BS EN 1999 - Design of Aluminium Structures. BSI, London, the UK.

British Standards Institution. Eurocode 1: BS EN 1991-2 - Actions on Structures. BSI, London, the UK.

British Standards Institution. BS EN ISO 3506-1 - Mechanical properties of corrosion-resistant stainless steel fasteners. Bolts, screws and studs. BSI, London, the UK.

British Standards Institution. BS5400: Steel Concrete and Composite Bridges. BSI, London, the UK.

Highways Agency. Design Manual for Roads and Bridges, BD49 - Design Rules for Aerodynamic Effects on Bridges. Highways Agency, London, the UK.

Highways Agency. Design Manual for Roads and Bridges, BD37 - Loads for Highway Bridges. Highways Agency, London, the UK.

Highways Agency. Design Manual for Roads and Bridges, BD29 - Design Criteria for Footbridges. Highways Agency, London, the UK.

Save International. Value Standard and Body of Knowledge (2007). SAVE, New Jersey, the US.

Cite this article as: Tzaveas T., "East Industrial Pedestrian Bridge: A Case Study of Value Engineering and High Performance Material", International Conference on Civil Infrastructure and Construction (CIC 2020), Doha, Qatar, 2-5 February 2020, DOI: https://doi.org/10.29117/cic.2020.0129 